\title{
Dermoscopy of Subungual Wart
}

\author{
Sweta Subhadarshani ${ }^{1}$, Jayati Sarangi ${ }^{2}$, Kaushal K. Verma ${ }^{1}$
}

1 Department of Dermatology and Venereology, All India Institute of Medical Sciences, New Delhi, India

2 Department of Pathology, All India Institute of Medical Sciences, New Delhi, India

Key words: dermoscopy, wart, nail, onychoscopy

Citation: Subhadarshani S, Sarangi J, Verma KK. Dermoscopy of subungual wart. Dermatol Pract Concept. 2019;9(1):22-23. DOI: https:// doi.org/10.5826/dpc.0901a06

Published: January 31, 2019

Copyright: (02019 Subhadarshani et al. This is an open-access article distributed under the terms of the Creative Commons Attribution License, which permits unrestricted use, distribution, and reproduction in any medium, provided the original author and source are credited.

Funding: None.

Competing interests: The authors have no conflicts of interest to disclose.

Authorship: All authors have contributed significantly to this publication.

Corresponding author: Sweta Subhadarshani, MD, Department of Dermatology and Venereology, All India Institute of Medical Sciences, Ansari Nagar, New Delhi 110049, India. Email: Shweta.aiims07@gmail.com

\section{Case Presentation}

A 26-year-old man presented with a 6-month history of distortion of the right thumbnail and crusting under the nail. On examination, there was yellowish discoloration of the nail along with subungual hyperkeratosis with hemorrhagic spots visible through the nail plate (Figure 1). Dermoscopy (Heine Delta 20T, polarized, 16x) of the nail plate showed yellowish white irregular discoloration of the nail bed along with broad, dark red clods suggestive of hemorrhage (Figure 2A), while that of the hyponychium showed yellowish brown keratotic areas with horizontal and vertical fractures, multiple dark red and black dots, some of which had white halo (Figure 2B). Histopathology showed hyperkeratosis, parakeratosis, papillomatosis, acanthosis, hemorrhage in the stratum corneum, and prominent keratohyalin granules compatible with a diagnosis of subungual wart.

\section{Conclusions}

Onychoscopy is a recent real-time diagnostic aid in the diagnosis of benign and malignant nail disorders and shows characteristic features of a specific dermatosis. Subungual

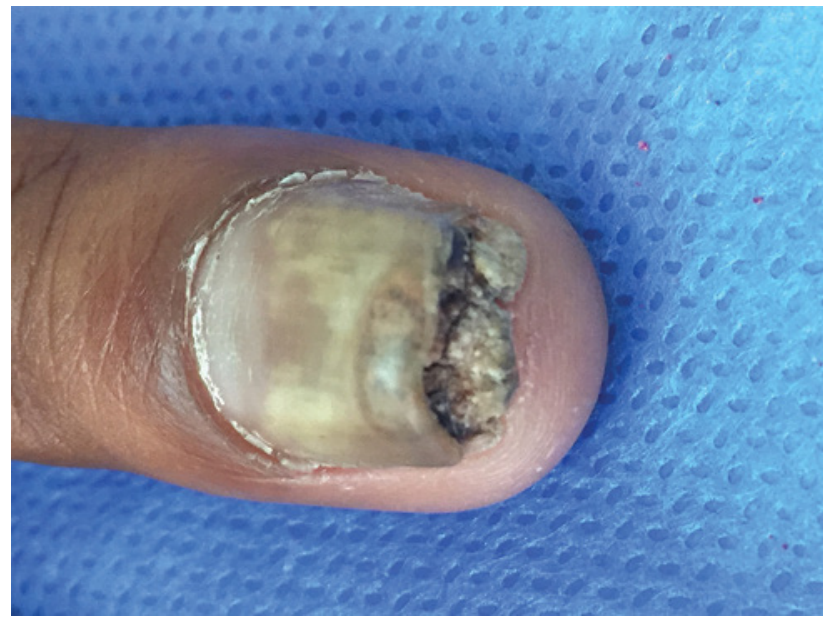

Figure 1. Yellowish discoloration of nail; subungual hyperkeratosis and hemorrhagic spots visible through nail plate. [Copyright: (O2019 Subhadarshani et al.]

wart can be seen on dermoscopy as thick adherent whiteyellow scales, fissures, and dotted dilated vessels (with intermingled hemorrhagic dots) which may be surrounded by a white halo [1,2]. It can be readily differentiated from other subungual pathologies on dermoscopy such as subungual exostosis or infective conditions such as onycho- 

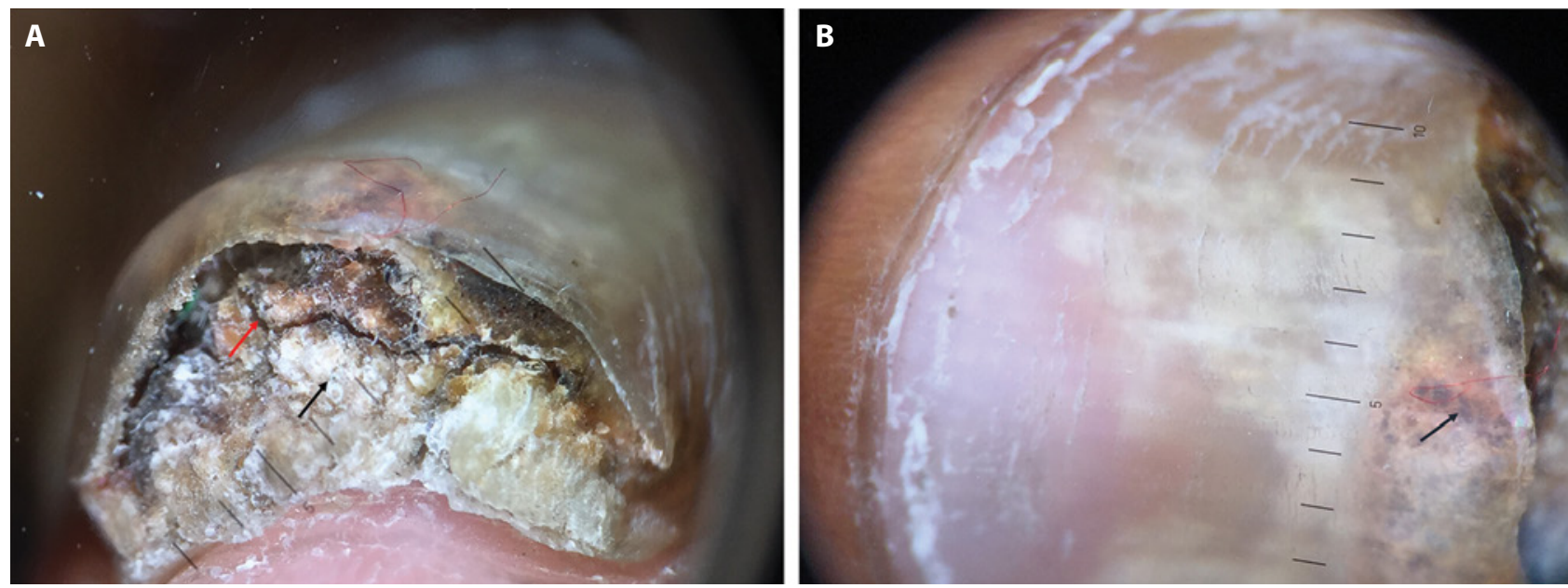

Figure 2. (A) Dermoscopy (Heine Delta 20T, polarized, 16x) of nail plate shows yellowish white irregular discoloration of nail bed along with broad, dark red clods (black arrow) suggestive of hemorrhage. (B) Dermoscopy (Heine Delta 20T, polarized, 16x) of hyponychium shows yellowish brown keratotic areas with horizontal and vertical fractures (red arrow), multiple dark red and black dots (black arrow), some of which have white halo. [Copyright: @2019 Subhadarshani et al.]

mycosis, which can result in subungual hyperkeratosis. Subungual exostosis shows hyperkeratosis, vascular ectasia, and ulceration, while onychomycosis shows a jagged edge of the proximal margin of the onycholytic area with sharp structures (spikes) directed to the proximal fold and aurora borealis pattern.

\section{References}

1. Piccolo V, Argenziano G, Alessandrini AM, Russo T, Starace M, Piraccini BM. Dermoscopy of subungual exostosis: a retrospective study of 10 patients. Dermatology. 2017;233(1):80-85.

2. Piraccini BM, Bruni F, Starace M. Dermoscopy of non-skin cancer nail disorders. Dermatol Ther. 2012;25(6): 594-602. 